\title{
Evolutionary divergence and functions of the human acyl-CoA thioesterase gene (ACOT) family
}

\author{
Chad Brocker,' Christopher Carpenter,' Daniel W. Nebert ${ }^{2 *}$ and Vasilis Vasiliou ${ }^{*}$ \\ 'Molecular Toxicology and Environmental Health Sciences Program, Department of Pharmaceutical Sciences, University of Colorado \\ Denver, Aurora, CO 80045, USA \\ ${ }^{2}$ Department of Environmental Health and Center for Environmental Genetics (CEG), University of Cincinnati Medical Center, \\ Cincinnati, OH 45267-0056, USA \\ *Correspondence to: E-mail: dan.nebert@uc.edu; vasilis.vasiliou@ucdenver.edu
}

Date received (in revised form): 25th July 2010

\begin{abstract}
The acyl-CoA thioesterase gene (ACOT) family encodes enzymes that catalyse the hydrolysis of acyl-CoA thioester compounds, also known as activated fatty acids, to their corresponding non-esterified (free) fatty acid and coenzyme $\mathrm{A}(\mathrm{CoASH})$. These enzymes play a very important role in lipid metabolism by maintaining cellular levels and proper ratios of free and activated fatty acids, as well as $\mathrm{CoASH}$. Within the acyl-CoA family there are two distinct subgroups, type I and type II. Despite catalysing the same reaction, the two groups are not structurally similar and do not share sequence homology, strongly suggesting convergent evolution. This suggestion is further supported if one compares the human with the mouse and rat ACOT gene families. To date, four human type I ACOTs have been identified which belong to the $\alpha / \beta$-hydrolase fold enzyme superfamily. Type II ACOTs fall into the 'hot dog' fold superfamily. There are currently six human type II genes; however, two homologous proteins, thioesterase superfamily members 4 (THEM4) and 5 (THEM5) share common type II structural features and, in the case of THEM4, acylCoA thioesterase activity - suggesting that the family may be larger than previously realised. Although recent studies have greatly expanded the current understanding of these proteins and their physiological importance, there are a number of members whose functions are relatively unexplored and which warrant further investigation.
\end{abstract}

Keywords: ACOT, acyl-CoA, thioesterase, hydrolase, gene family, evolution, human genome

\section{Introduction}

Acyl-CoA thioesterases (ACOTs) represent a group of enzymes that metabolise acyl-CoA esters to their corresponding non-esterified fatty acid and coenzyme A (CoASH). In addition to 'acyl-CoA thioesterases', these enzymes also have been described as 'acyl-CoA hydrolases', 'acyl-CoA thioester hydrolases' and 'deacylases'. The reaction catalysed by ACOT enzymes is very important during fatty acid metabolism. As such, ACOT genes are found throughout prokaryotic and eukaryotic organisms. In mammals, acyl-CoA thioesterase expression is widespread and activity is detectable within many tissues and cell types. ACOT activity helps to regulate cellular pools, as well as proper ratios, of activated fatty acyl-CoAs (acyl-CoA esters), free fatty acids (non-esterified fatty acids) and CoASH.

These enzymes are found within a number of subcellular locations, including peroxisomes, mitochondria and the cytosol. Peroxisomal and mitochondrial ACOTs are thought to play a major role in fatty acid degradation via $\beta$-oxidation, which provides energy through the citric acid cycle, as 
well as in ketone body formation. In order for a fatty acid to be transported into the peroxisome for $\beta$-oxidation, it must first be activated to its coenzyme A ester by acyl-CoA synthetases. Some acyl-CoA esters undergo $\beta$-oxidation much more slowly than others — which can effectively sequester large amounts of CoASH and limit the activity of acyl-CoA synthetases and the rate of subsequent fatty acid $\beta$-oxidation. Under these conditions, ACOTs may act to increase the levels of free $\mathrm{CoASH}$, thereby restoring fatty acid $\beta$-oxidation. ${ }^{1}$ Mammalian ACOTs are capable of metabolising a wide variety of compounds, including short-, medium- and long-chain acyl-CoAs. They can also metabolise polyunsaturated fatty acyl-CoAs, branched-chain acyl-CoAs and aromatic-acylCoAs. $^{2}$ These compounds are not only utilised during $\beta$-oxidation and fatty acid degradation, but also have roles in inflammation, ion channel opening, signal transduction, lipid biosynthesis and gene regulation. ${ }^{2,3}$

Ten human ACOT genes have been identified to date, compared with 13 in mice and 12 in rats. These genes can be divided into two groups, based upon differences in sequence and structure. The first group includes type I ACOT proteins. These enzymes are members of the $\alpha / \beta$-hydrolase fold enzyme superfamily, which includes other esterase-activity-exhibiting enzymes such as carboxylesterases and lipases.

Type II ACOTs make up the second group; these enzymes are members of the 'hot dog' fold enzyme superfamily, which includes a wide variety of functionally diverse proteins. Despite catalysing the same reaction, type I and type II enzymes share no similarity in terms of structure or sequence indicating that the two groups are analogous and not homologous. This observation is reminiscent of the cytochrome P450 (CYP) and nitric oxide synthase (NOS) gene families. Studies with purified NOS proteins revealed that they possess P450-like activity; however, detailed alignment analyses showed $<15$ per cent amino acid identity, thereby confirming that these two gene families were not evolutionarily related. ${ }^{4}$ It was thus concluded that this was an example of convergent evolution. In other words, during the evolution of enzyme activities needed at a particular subcellular location (probably more than 1 billion years ago) Mother Nature needed a 'P450-like activity' and assigned that task to the nearest protein, which happened to be an NOS. The same thing most likely happened to the type I versus type II ACOTs.

The revised nomenclature for human, mouse and rat acyl-CoA thioesterase genes was established by Hunt et al. and has been accepted by the HUGO Gene Nomenclature Committee. ${ }^{5}$ In recent years, a number of human ACOTs have been recombinantly expressed and enzymatically characterised, although the exact physiological function and importance of many of these enzymes remain unknown.

\section{Human ACOT genes and evolution}

To date, a total of ten genes have been identified within the human genome, all of which exhibit acyl-CoA thioesterase activity (Table 1). These ten genes can be sub-divided into two distinct groups, type I and type II, based on sequence and structural homology. Type I ACOTs comprise the smaller group, which contains only four genes: ACOT1, ACOT2, ACOT4 and ACOT6. The larger group includes six genes, and these members are referred to as type II. There is no sequence homology between the two groups, and the translated proteins have very different domain architecture, indicating two distinct, evolutionarily unrelated, origins (Figure 1) and, most likely, an example of convergent evolution.

The type I proteins, ACOT1, ACOT2, ACOT4 and ACOT6, share a high degree of sequence homology (Table 2). ACOT1 and ACOT2 are the most closely related, having 98 per cent amino acid sequence identity. ACOT4 is 70 per cent identical to both ACOT1 and ACOT2. ACOT6 is the least homologous, but still shares 54-57 per cent sequence identity with the other members. None of these proteins shares any significant sequence homology with the type II proteins. The four type I genes form an 80 kilobase gene cluster on chromosome $14 \mathrm{q} 24.3$, indicating that they most likely have arisen as the result of gene duplication. 
Table I. List of all human (ACOT) with full gene name, aliases, chromosomal location, isoforms, NCBI RefSeq mRNA accession number, $\mathrm{NCBI}$ RefSeq protein accession number and total amino acid number (AA\#)

\begin{tabular}{|c|c|c|c|c|c|c|c|}
\hline Gene & $\begin{array}{l}\text { Full gene } \\
\text { name }\end{array}$ & Aliases & Chromosome & Isoforms & Ref mRNA & Ref protein & AA\# \\
\hline ACOTI & $\begin{array}{l}\text { Acyl-CoA } \\
\text { thioesterase I }\end{array}$ & $\begin{array}{l}\text { ACH2, } \\
\text { CTE-I, } \\
\text { LACH2 }\end{array}$ & $14 q 24.3$ & & NM00I037I6I.I & NP00I032238 & 421 \\
\hline ACOT2 & $\begin{array}{l}\text { Acyl-CoA } \\
\text { thioesterase } 2\end{array}$ & $\begin{array}{l}\text { MTEI, PTE2, } \\
\text { CTEIA, } \\
\text { PTE2A, } \\
\text { ZAPI } 28\end{array}$ & $14 q 24.3$ & & NM00682I.4 & NP0068I 2.3 & 483 \\
\hline ACOT4 & $\begin{array}{l}\text { Acyl-CoA } \\
\text { thioesterase } 4\end{array}$ & $\begin{array}{l}\text { FLJ3 I } 235, \\
\text { PTE-Ib, } \\
\text { PTEIB, } \\
\text { PTE2B }\end{array}$ & $14 q 24.3$ & & NMI5233I.3 & NP689544.3 & 421 \\
\hline АСОT6 & $\begin{array}{l}\text { Acyl-CoA } \\
\text { thioesterase } 6\end{array}$ & $\begin{array}{l}\text { Cl4orf42, } \\
\text { cl4_5530, } \\
\text { MGCI6I656, } \\
\text { MGCI6I658 }\end{array}$ & $14 q 24.3$ & & NM00I037I62.I & NP00I032239.I & 207 \\
\hline \multirow[t]{4}{*}{ ACOT7 } & $\begin{array}{l}\text { Acyl-CoA } \\
\text { thioesterase } 7\end{array}$ & $\begin{array}{l}\text { BACH, } \\
\text { ACHI, ACT, } \\
\text { CTE-II, } \\
\text { LACH, } \\
\text { LACHI, } \\
\text { MGCII } 26\end{array}$ & $|p 36.3|-p 36.1 \mid$ & hBACHa & NM007274.3 & NP009205.3 & 370 \\
\hline & & & & $\mathrm{hBACHb}$ & NMI8I864.2 & NP863654.I & 380 \\
\hline & & & & $\mathrm{hBACHc}$ & NMI8I865.2 & NP863655.I & 350 \\
\hline & & & & hBACHd & NMI8I866.2 & NP863656.I & 329 \\
\hline АСОT8 & $\begin{array}{l}\text { Acyl-CoA } \\
\text { thioesterase } 8\end{array}$ & $\begin{array}{l}\text { hTE, PTEI, } \\
\text { PTE2, PTE-2, } \\
\text { HNAACTE, } \\
\text { hACTE-III }\end{array}$ & $20 q 13.12$ & & NM005469.2 & NP005460.2 & 319 \\
\hline \multirow[t]{2}{*}{ ACOT9 } & $\begin{array}{l}\text { Acyl-CoA } \\
\text { thioesterase } 9\end{array}$ & $\begin{array}{l}\text { CGI-16, } \\
\text { MT-ACT48, } \\
\text { ACATE2 }\end{array}$ & Xp22.II & Isoform a & NM00I037I7I.I & NP00I032248.I & 448 \\
\hline & & & & Isoform b & NM00I033583.2 & NP00I028755.2 & 439 \\
\hline \multirow[t]{2}{*}{ АСОТII } & $\begin{array}{l}\text { Acyl-CoA } \\
\text { thioesterase II }\end{array}$ & $\begin{array}{l}\text { BFIT, THEA, } \\
\text { BFITI, BFIT2, } \\
\text { THEMI, } \\
\text { STARDI4, } \\
\text { KIAA0707 }\end{array}$ & Ip32.3 & BFITI & NM0I5547.2 & NP056362.I & 607 \\
\hline & & & & BFIT2 & NMI47I6I.2 & NP67I5I7.I & 594 \\
\hline
\end{tabular}


Table I. Continued

\begin{tabular}{|c|c|c|c|c|c|c|c|}
\hline Gene & $\begin{array}{l}\text { Full gene } \\
\text { name }\end{array}$ & Aliases & Chromosome & Isoforms & Ref mRNA & Ref protein & AA\# \\
\hline ACOTI 2 & $\begin{array}{l}\text { Acyl-CoA } \\
\text { thioesterase } 12\end{array}$ & $\begin{array}{l}\text { Cach, } \\
\text { THEAL, } \\
\text { CACH-I, } \\
\text { STARDI5, } \\
\text { MGCI05II4 }\end{array}$ & $5 q 14.1$ & & NMI 30767.2 & NP570I23.I & 555 \\
\hline \multirow[t]{2}{*}{ АСОТ I 3} & $\begin{array}{l}\text { Acyl-CoA } \\
\text { thioesterase } 13\end{array}$ & $\begin{array}{l}\text { THEM2, } \\
\text { HTOI2, } \\
\text { MGC496I, } \\
\text { PNAS-27 }\end{array}$ & $6 p 22.3$ & Isoform I & NM0I8473.3 & NP060943.I & 140 \\
\hline & & & & Isoform 2 & NM0 18473.3 & NP060943.I & 117 \\
\hline THEM4 & $\begin{array}{l}\text { Thioesterase } \\
\text { superfamily } \\
\text { member } 4\end{array}$ & $\begin{array}{l}\text { CTMP, } \\
\text { MGC29636 }\end{array}$ & $|q 2|$ & & NM053055.3 & NP444283.2 & 240 \\
\hline THEM5 & $\begin{array}{l}\text { Thioesterase } \\
\text { superfamily } \\
\text { member } 5\end{array}$ & $\begin{array}{l}\text { FLJ37964; } \\
\text { MGCI } 26659 ; \\
\text { MGCI } 38444\end{array}$ & |q21.3 & & NMI 82578.3 & NP872384.I & 247 \\
\hline
\end{tabular}

A similar situation is observed within the mouse and rat orthologues $A \cot 1, A \cot 2, A \cot 3, A \cot 4$, $A \cot 5$ and $A \cot 6$, which are clustered on chromosomes 12 D3 and 6q31, respectively. A cladogram depicting the evolutionary relationships observed between human, mouse and rat type I proteins is shown in Figure 2A. This cladogram clearly shows that, prior to the mammalian radiation approximately 75 million years ago (MYA), there was a single ancestral ACOT1 (or -2) gene; after the mammalian radiation but before the mouse-rat split at approximately 15 MYA, this single ancestor divided twice to become four rodent Acot functional genes (now named $A \cot 1, A \cot 3, A \cot 4$ and Acot5). By contrast, after the mammalian radiation, the human single ancestor duplicated into a second functioning gene, leading to ACOT1 and ACOT2. On the other hand, ACOT4 and ACOT6 existed before the mammalian radiation and remain conserved to this day in human, mouse and rat.

In the mouse, the ACOT3, ACOT4 and ACOT5 enzymes each exhibit specific expression patterns and enzyme substrate specificity. Human ACOT4 carries out the reactions catalysed by all three of these mouse enzymes. The lack of a human orthologue to mouse
$A \cot 3$ and $A \cot 5$ was suggested to be the result of convergent evolution; it was hypothesised that convergent, functional evolution led to a reduced need for multiple enzymes in humans. ${ }^{6}$ The Protein Basic Local Alignment Search Tool (BLASTP) analysis of all non-redundant protein sequences, however, failed to identify orthologues for ACOT3 or ACOT5 in non-rodent species. This finding suggests that ACOT4 was an ancestral gene encoding an ACOT4 enzyme with broad substrate recognition, and that gene duplication events led to ACOT1 (or -2), and that further duplication events led to the mouse $A \cot 3$ and $A$ cot 5 genes, encoding the ACOT3 and ACOT5 enzymes, which have similar substrate specificity to that of mouse ACOT4 and human ACOT4.

Differences exist among the human, mouse and rat ACOT6 orthologues. Analysis of the genomic ACOT6 sequence indicates transcription of a complete mRNA containing three exons; however, a full-length mRNA is not detectable. ${ }^{6}$ A shorter transcript, in which the first exon was omitted, was identified. Subsequent translation begins at a methionine located within the second exon, producing a truncated protein that lacks the $\mathrm{N}$-terminal acyl-CoA thioester hydrolase domain found within 


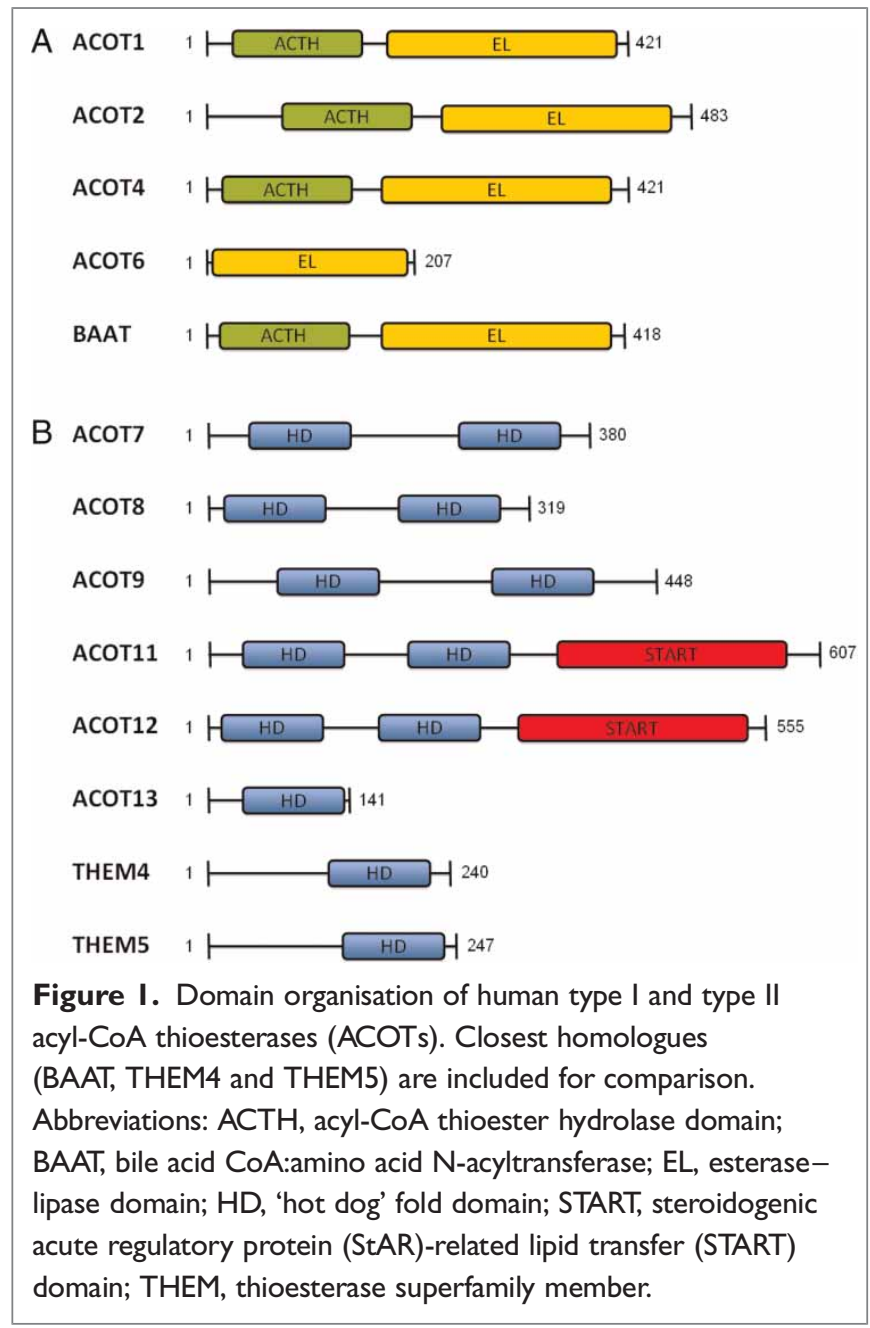

other type I proteins. The truncated protein contains all residues required for catalysis and could therefore still produce an active acyl-CoA thioesterase. This is not true for the mouse or rat ACOT.

The BLAST results indicate that bile acid CoA:amino acid N-acyltransferase (BAAT) is the only enzyme to share significant sequence homology with type I ACOTs. BAAT shares 45 per cent sequence identity with ACOT1, ACOT2 and ACOT4. ACOT6 shares 37 per cent sequence identity with BAAT. BAAT is known to participate in the transfer of bile acids from the acyl-CoA thioester to either glycine or taurine, which are used for bile salt secretion. BAAT contains the same domain architecture and ACOT1, ACOT2 and ACOT4; however, the catalytic triad found within the esterase-lipase domain contains a cysteine, instead of the highly conserved serine residue. This substitution may explain the difference in activity (ie transferase versus hydrolase) observed between the two enzymes. Despite sharing catalytic domains, other $\alpha / \beta$-hydrolase fold enzyme superfamily esterases, such as carboxylesterase 1 (CES1) and acetylcholinesterase (ACHE), share very little sequence homology with their acyl-CoA thioesterase relatives. CES1 has thus been added as an evolutionarily unrelated gene to Figure 2A simply to 'ground' the dendrogram.

Type II ACOTs are strikingly less related than type I proteins. None of the type II proteins shares more than 25 per cent identity and many do not share any significant sequence similarity (Table 2). Unlike the type I genes, type II ACOTs are widely distributed throughout the genome. With the exception of ACOT13, all type II proteins contain double 'hot dog' domains, which may have evolved as a gene duplication event that allowed for the accommodation of bulky substrates. ${ }^{7}$ ACOT7 shares 19 per cent identity with ACOT9, 22 per cent with ACOT11 and 24 per cent identity with ACOT12. ACOT9 shares 22 per cent identity with ACOT12 but displays no homology with ACOT11. ACOT8 has no significant sequence homology with any of the other type II proteins. ACOT11 and ACOT12 share 51 per cent sequence identity and both enzymes contain a C-terminal steroidogenic acute regulatory protein (StAR)related lipid transfer (START) domain, which seems to be specific to mammalian evolution. ${ }^{7}$

The human genome contains 15 START-containing proteins, including ACOT11 and ACOT12. ${ }^{8}$ The $\mathrm{N}$-terminal region of both ACOTs is unrelated to any of the other START-containing enzymes, suggesting that these proteins may be the result of a gene fusion event between an acyl-thioesterase and START domain-containing sequences. Like ACOT8, ACOT13 shares no significant sequence similarity to the other type II proteins. BLAST results did identify 22 per cent sequence identity with thioesterase superfamily member 4 (THEM4 is the official name) - also known as Akt C-terminal modulator protein (CTMP). This small degree of homology suggests that the two proteins may share common evolutionary ties. Another 
Table 2. Amino acid sequence identity between type I and type II human acyl-CoA thioesterases (ACOTs). Identity was determined using BLASTP and non-redundant human protein sequences. The predominant isoform sequences were used for ACOTs having multiple protein variants. Homology was considered not significant and left blank if the observed identity was less than 15 per cent

\begin{tabular}{|c|c|c|c|c|c|c|c|c|}
\hline \multicolumn{9}{|c|}{ Type I ACOTs } \\
\hline & ACOTI & АСОТ 2 & АСОТ4 & АСОТ6 & BAAT & & & \\
\hline \multicolumn{9}{|l|}{ ACOTI } \\
\hline АСОТ2 & $98 \%$ & & & & & & & \\
\hline ACOT4 & $70 \%$ & $70 \%$ & & & & & & \\
\hline АСОT6 & $57 \%$ & $57 \%$ & $54 \%$ & & & & & \\
\hline BAAT & $45 \%$ & $45 \%$ & $45 \%$ & $37 \%$ & & & & \\
\hline \multicolumn{9}{|c|}{ Type II ACOTs } \\
\hline & АСОT7 & АСОТ8 & АСОТ9 & ACOTII & ACOTI 2 & АСОТ I3 & THEM4 & THEM5 \\
\hline \multicolumn{9}{|l|}{ ACOT7 } \\
\hline АСОT8 & - & & & & & & & \\
\hline АСОТ9 & $19 \%$ & - & & & & & & \\
\hline ACOTII & $22 \%$ & - & - & & & & & \\
\hline АСОTI2 & $24 \%$ & - & $22 \%$ & $51 \%$ & & & & \\
\hline АСОTI3 & - & - & - & - & - & & & \\
\hline THEM4 & - & - & - & - & - & $22 \%$ & & \\
\hline THEM5 & - & - & - & - & - & - & $\%$ & \\
\hline
\end{tabular}

Abbreviations: BAAT, bile acid CoA:amino acid $\mathrm{N}$-acyltransferase; THEM, thioesterase superfamily member

protein, thioesterase superfamily member 5 (THEM5) shares more than 37 per cent identity with THEM4 but lacks significant sequence homology with ACOT13. Similar to ACOT13, both THEM4 and THEM5 contain a single 'hot dog' domain. Furthermore, type II proteins, THEM4 and THEM5 do not share sequence homology with other 'hot dog' domain superfamily members. The 'hot dog' fold-containing proteins possess highly conserved structural elements that are created by highly divergent sequences. ${ }^{9}$ This high degree of divergence makes evolutionary comparisons difficult without three-dimensional structures, because conservation of structural interactions is not directly associated with residue conservation.

BLAST results indicate that mammalian type II ACOTs are evolutionarily conserved. A cladogram depicting the evolutionary relationships observed between human, mouse and rat type II protein is shown in Figure 2B. The mouse genome contains an additional type II ACOT, $A \cot 10$, which shares approximately 95 per cent mRNA nucleotide identity with ACOT9. ${ }^{10}$ Unlike ACOT3 and ACOT5, however, which are found in both mouse and rat, ACOT10 has only been identified in mice, indicating that the gene duplication event occurred less than 17 MYA - that is, after the mouse-rat split. In mouse, $A \cot 9$ is located at chromosomal location X F3 and $A \cot 10$ is found on 15 A2. The amplification and movement of genes to a new chromosomal location is often facilitated by transposable elements, as opposed to duplications occurring within close proximity on a chromosome, which are attributed to errors during meiosis. ${ }^{11}$ The remaining seven 


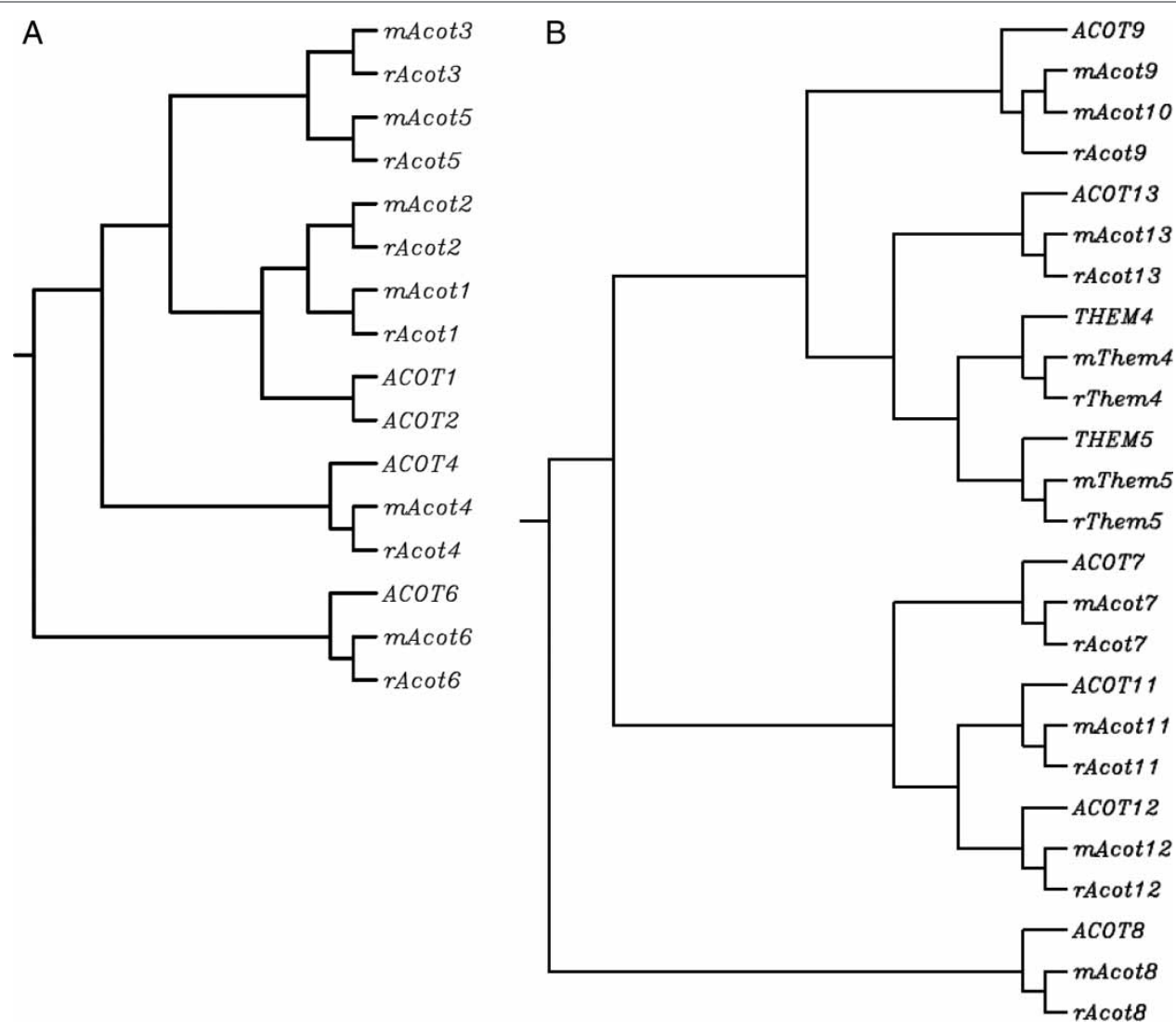

Figure 2. Acyl-CoA thioesterase (ACOT) type I and type II cladograms. Cladograms depicting the evolutionary relationships between human, mouse $(m)$ and rat $(r)$ orthologous protein sequences for type I (A) and type II (B) ACOTs. Phylogenetic trees were created using ClustalW2. Human carboxylesterase I (CESI) is an unrelated esterase that was added simply to 'ground' the tree.

type II ACOT genes are highly conserved among human, mouse and rat, confirming that these genes were all present in the ancestor prior to the mammalian radiation.

Type I and type II acyl-CoA thioesterases represent two structurally distinct enzyme groups. These groups catalyse the same reaction but have distinct evolutionary origins and reaction mechanisms. An unrooted dendrogram - created using protein sequences from both type I and type II enzymes, closest-related homologues (BAAT, THEM4 and THEM5), distant $\alpha / \beta$-hydrolase fold homologues (CES1, ACHE) and $\beta$-actin (ACTB) as an unrelated sequence - exemplifies how dissimilar the two groups are from one another (Figure 3). It also illustrates how the type II proteins must have originated from a common, evolutionarily distant, predecessor (as reflected in individual branch proximity to the origin of the tree).

\section{Type I ACOT structures}

Type I ACOTs share a high degree of sequence and structural homology. ACOT1, ACOT2 and ACOT4 contain an N-terminal acyl-CoA thioester hydrolase domain (Pfam 04775). This domain does not participate directly in catalysis, but studies indicate that the N-terminus plays an important role in regulating enzyme activity. ${ }^{3}$ All type I proteins also contain a C-terminus esterase-lipase superfamily domain. All residues required for catalysis are located within this domain, which is also known as the $\alpha / \beta$-hydrolase fold.

Proteins that share this common structural element make up the functionally diverse $\alpha / \beta$-hydrolase fold superfamily of enzymes. Within this family are a number of other distantly related proteins that exhibit esterase activity, including carboxylesterases, ACHE and lipases. The superfamily has diverged greatly and 


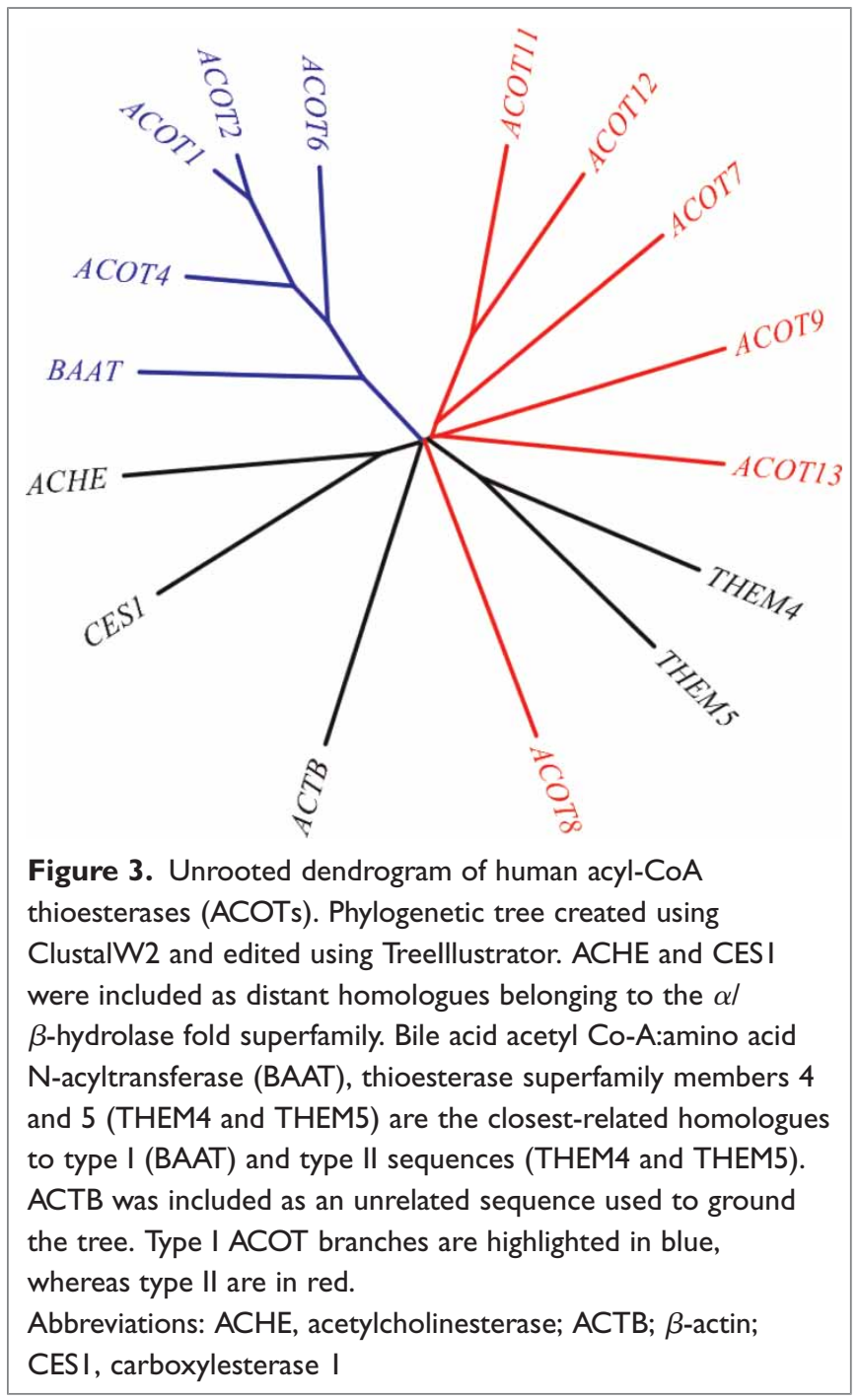

many members share very little sequence homology but are considered evolutionarily linked, based on structural homology. The enzyme activity is dependent on a catalytic triad composed of highly conserved serine, histidine and aspartate residues found within the active site. ${ }^{12}$ These three residues are conserved throughout type I ACOTs.

The crystalline structure for ACOT2 indicates that the enzyme is most likely biologically active as a monomer. ${ }^{13}$ The structure also reveals that the N-terminal acyl-CoA thioester hydrolase domain is connected to the active site by a long loop; it is postulated that this contributes substrate specificity, although this has yet to be verified experimentally. ${ }^{3}$ The four human type I ACOT genes are located on chromosome 14q24.3. ACOT1, ACOT2 and
ACOT3 each encode a transcript containing three exons. Genomic analysis suggests that ACOT6 should encode a similar 3 exon transcript; however, the only detectable transcript is truncated and utilises a start codon located within the $3^{\prime}$ end of exon two, resulting in the synthesis of a truncated protein. ${ }^{6}$ In addition to the four protein-coding genes, there is one intronless pseudogene, referred to as ACOT4P, located on chromosome $19 \mathrm{q} 13.12$. ACOTP4 is most likely the result of retroviral action that has inserted this DNA segment elsewhere in the genome. Attempts to translate the pseudogene results in numerous in-frame stop codons.

ACOT1, ACOT2 and ACOT4 have distinct subcellular localisation patterns and substrate specificity. ACOT1 is cytosolic and primarily responsible for the metabolism of long-chain acyl-CoAs. ${ }^{1,14}$ ACOT2 and ACOT1 can metabolise a similar range of substrates; however, ACOT2 contains an $\mathrm{N}$-terminal mitochondrial targeting signal which directs its transport to mitochondria. ${ }^{6}$ ACOT4 contains a C-terminal peroxisomal targeting signal, directing transport to peroxisomes, where it exhibits high activity against succinyl-CoA, as well as a wide variety of short-chain dicarboxylic-CoAs. ACOT4 can also metabolise medium- and longchain saturated- and unsaturated-CoAs. ${ }^{6,15}$ As previously mentioned, ACOT6 lacks the $\mathrm{N}$-terminal acyl-CoA thioester hydrolase domain.

Although studies have yet to confirm the enzymatic activity of ACOT6, the fact that it contains a complete C-terminal esterase-lipase domain supports its role as a functional esterase. ${ }^{13}$ ACOT6 is predicted to be cytosolic, due to a missense mutation which alters the peroxisomal targeting signal that is found in the $A \cot 6$ mouse ortholog. ${ }^{6}$ The subcellular localisation and substrate specificity for each enzyme suggest that each plays an important, non-redundant function within the cell.

\section{Type II ACOT structures}

The type II enzymes are more distantly related than type II ACOTs, as indicated by the relatively low degree of sequence conservation observed between proteins. Despite the low degree of sequence 
homology, the enzymes are structurally related and capable of metabolising similar substrates. ACOT7, formerly called brain acyl-CoA hydrolase (BACH), has been extensively characterised, owing to recent studies that indicate arachidonoyl-CoA as a preferred substrate. The metabolism of arachidonoyl-CoA by ACOT7 gives rise to arachidonic acid and coenzyme A. Arachidonic acid serves as a precursor for a number of compounds associated with inflammation, including prostaglandins, leukotrienes and thromboxanes. $^{16,17}$ ACOT7 also efficiently metabolises a number of long-chain-acyl-CoAs. ${ }^{18}$ ACOT8 is found in peroxisomes, where it metabolises a diverse range of acyl-CoA compounds. ${ }^{1}$ Substrates include short-, medium- and long-chain acyl-CoAs. ACOT8 also has high activity towards branched- and methylbranched chain acyl-CoAs.

ACOT9 contains a mitochondrial-targeting signal and metabolises medium- to long-chain acyl-CoAs. ACOT9 exhibits the highest activity towards myristoyl-CoA, which can then be used by $\mathrm{N}$-myristoyltransferase to modify proteins posttranslationally. ${ }^{10}$ Expression of mouse ACOT11, formerly referred to as brown fat-inducible thioesterase (BFIT), increases after cold shock. This protein was found to play a role in regulating thermogenesis, which helps to maintain body temperature. ${ }^{19}$ The human homologue, ACOT11, may be a risk factor for obesity. ${ }^{19}$ As previously mentioned, ACOT11 and ACOT12 contain a C-terminal START domain. The START domain in animals is commonly found within homeodomain transcription factors, where lipid-binding regulates transcription. $^{20}$ The binding of lipids to the START domain in ACOT11 or ACOT12 acts as a mechanism for regulating activity and for allowing for rapid enzyme activation. The kinetic properties of ACOT11 have yet to be determined. ACOT12 has highest activity towards acetyl-CoA. ${ }^{21}$

ACOT13, previously called thioesterase superfamily member 2 (THEM2), differs from the other type II ACOTs, in that it contains a single 'hot dog' domain. Immunocytochemistry identified ACOT13 within the cytosol and nucleus. ${ }^{22}$ Another study indicated that the enzyme is mitochondrial. ${ }^{23}$ The crystal structure and substrate specificity were recently determined using recombinant protein; results indicate that ACOT13 prefers medium- to long-chain-acyl-CoA thioesters. ${ }^{24}$ Interestingly, ACOT13 interacts with a START domain-containing protein named StarD2. The addition of StarD2 decreases the $\mathrm{K}_{\mathrm{cat}}$ of ACOT13 for a number of substrates. $^{25}$ This finding suggests that the START domains, identified in ACOT11 and ACOT12, could have a similar effect on enzyme activity. BLAST results indicate $>22$ per cent sequence identity between ACOT13 and THEM4.

Two separate studies have revealed that THEM4 is a mitochondrial protein. ${ }^{26,27}$ Recent in vitro kinetic activity studies revealed that THEM4 is a broad-range, high-activity acyl-CoA thioesterase. ${ }^{28}$ Similar to ACOT13, THEM4 preferentially metabolises medium- to long-chain acyl-CoA thioesters. As such, we recommend that the enzyme be renamed ACOT14 according to the accepted nomenclature established by Hunt et al. ${ }^{5}$ Acyl-CoA thioesterase activity has not been verified for THEM5; however, activity is probably based on the high degree of sequence identity with THEM4, but the exact role that this protein might play in fatty acid metabolism has yet to be determined.

\section{Conclusions}

The human acyl-CoA gene family represents a diverse group of enzymes that catalyse the hydrolysis of acyl-CoA thioesters to their corresponding free fatty acids and coenzyme A. These enzymes are involved in a number of cellular processes but are primarily thought to play an important role in lipid metabolism. Despite catalysing the same enzymatic reaction, the acyl-CoA thioesterase family members can be divided into two distinct groups, type I and type II. The two groups do not share common structural elements or sequence homology and are therefore considered analogous, rather than homologous, enzymes; this is probably another example of convergent evolution.

Type I proteins belong to the $\alpha / \beta$-hydrolase fold superfamily, whereas type II proteins fall into the "hot dog' fold superfamily. To date, four type I and six type II genes have been identified within the human 
genome. There are two additional genes, THEM4 and THEM5, which gene products share both structural and sequence homology with other type II proteins. THEM4 was identified as having acyl-CoA thioesterase activity and should be considered as a new family member. In the future, perhaps THEM5 should also be considered as a type II ACOT family member. Recent studies have greatly expanded the current understanding of acyl-CoA thioesterases; however, many precise physiological functions are not completely understood and warrant further investigation.

\section{Acknowledgments}

We would like to thank our colleagues for valuable discussion and critically reviewing this manuscript. This work was supported, in part, by the following NIH grants; R01EY17963 and R21AA017754 (V.V.) and P30ES06096 (D.W.N.).

\section{References}

1. Hunt, M.C., Solaas, K., Kase, B.F. and Alexson, S.E. (2002), 'Characterization of an acyl-coA thioesterase that functions as a major regulator of peroxisomal lipid metabolism', J. Biol. Chem. Vol. 277, No. 2, pp. $1128-1138$.

2. Hunt, M.C. and Alexson, S.E. (2008), 'Novel functions of acyl-CoA thioesterases and acyltransferases as auxiliary enzymes in peroxisomal lipid metabolism', Prog. Lipid Res. Vol. 47, No. 6, pp. 405-421.

3. Kirkby, B., Roman, N., Kobe, B. et al. (2010), 'Functional and structural properties of mammalian acyl-coenzyme A thioesterases', Prog. Lipid Res.

4. Nelson, D.R., Kamataki, T., Waxman, D.J., Guengerich, F.P. et al. (1993), 'The P450 superfamily: Update on new sequences, gene mapping, accession numbers, early trivial names of enzymes, and nomenclature', DNA Cell Biol. Vol. 12, Jan/Feb, pp. 1-51.

5. Hunt, M.C., Yamada, J., Maltais, L.J., Wright, M.W. et al. (2005), 'A revised nomenclature for mammalian acyl-CoA thioesterases/hydrolases', J. Lipid Res. Vol. 46, No. 9, pp. 2029-2032.

6. Hunt, M.C., Rautanen, A., Westin, M.A., Svensson, L.T. et al. (2006), 'Analysis of the mouse and human acyl-CoA thioesterase (ACOT) gene clusters shows that convergent, functional evolution results in a reduced number of human peroxisomal ACOTs', FASEB J. Vol. 20, No. 11, pp. $1855-1864$

7. Dillon, S.C. and Bateman, A. (2004), 'The Hotdog fold: Wrapping up a superfamily of thioesterases and dehydratases', BMC. Bioinformatics Vol. 5, p. 109.

8. Alpy, F. and Tomasetto, C. (2005), 'Give lipids a START: The StAR-related lipid transfer (START) domain in mammals', J. Cell Sci. Vol. 118, No. 13, pp. 2791-2801.

9. Pidugu, L.S., Maity, K., Ramaswamy, K., Surolia, N. et al. (2009), 'Analysis of proteins with the 'hot dog' fold: Prediction of function and identification of catalytic residues of hypothetical proteins', BMC Struct. Biol. Vol. 9, p. 37.

10. Poupon, V., Begue, B., Gagnon, J., Dautry-Varsat, A. et al. (1999), 'Molecular cloning and characterization of MT-ACT48, a novel mitochondrial acyl-CoA thioesterase', J. Biol. Chem. Vol. 274, No. 27, pp. 19188-19194.
11. De Grassi, A., Lanave, C. and Saccone, C. (2008), 'Genome duplication and gene-family evolution: The case of three OXPHOS gene families', Gene Vol. 421, No. 1-2, pp. 1-6.

12. Li, J.J. and Bugg, T.D. (2007), 'Investigation of a general base mechanism for ester hydrolysis in C-C hydrolase enzymes of the alpha/betahydrolase superfamily: A novel mechanism for the serine catalytic triad', Org. Biomol. Chem. Vol. 5, No. 3, pp. 507-513.

13. Mandel, C.R., Tweel, B. and Tong, L. (2009), 'Crystal structure of human mitochondrial acyl-CoA thioesterase (ACOT2)', Biochem. Biophys. Res. Commun. Vol. 385, No. 4, pp. 630-633.

14. Dongol, B., Shah, Y., Kim, I., Gonzalez, FJ. et al. (2007), 'The acyl-CoA thioesterase I is regulated by PPARalpha and HNF4alpha via a distal response element in the promoter', J. Lipid Res. Vol. 48, pp. $1781-1791$.

15. Westin, M.A., Hunt, M.C. and Alexson, S.E. (2005), 'The identification of a succinyl-CoA thioesterase suggests a novel pathway for succinate production in peroxisomes', J. Biol. Chem. Vol. 280, pp. 38125-38132.

16. Rouzer, C.A., Ivanova, P.T., Byrne, M.O., Milne, S.B. et al. (2006), 'Lipid profiling reveals arachidonate deficiency in RAW264.7 cells: Structural and functional implications', Biochemistry Vol. 45, pp. $14795-14808$.

17. Nebert, D.W. and Karp, C.L. (2008), 'Endogenous functions of the aryl hydrocarbon receptor (AHR): Intersection of cytochrome P450 1 (CYP1)-metabolized eicosanoids and AHR biology', J. Biol. Chem. Vol. 283, No. 52, pp. 36061-36065.

18. Yamada, J., Kurata, A., Hirata, M., Taniguchi, T. et al. (1999), 'Purification, molecular cloning, and genomic organization of human brain long-chain acyl-CoA hydrolase', J. Biochem. Vol. 126, No. 6, pp. 1013-1019.

19. Adams, S.H., Chui, C., Schilbach, S.L., Yu, X.X. et al. (2001), 'BFIT, a unique acyl-CoA thioesterase induced in thermogenic brown adipose tissue: cloning, organization of the human gene and assessment of a potential link to obesity', Biochem. J. Vol. 360, No. 1, pp. 135-142.

20. Schrick, K., Nguyen, D., Karlowski, W.M. and Mayer, K.F. (2004), 'START lipid/sterol-binding domains are amplified in plants and are predominantly associated with homeodomain transcription factors', Genome Biol. Vol. 5, No. 6, pp. R41.

21. Suematsu, N. and Isohashi, F. (2006), 'Molecular cloning and functional expression of human cytosolic acetyl-CoA hydrolase', Acta Biochim. Pol. Vol. 53, No. 3, pp. 553-561.

22. Cheng, Z., Bao, S., Shan, X., Xu, H. et al. (2006), 'Human thioesterase superfamily member 2 (hTHEM2) is co-localized with beta-tubulin onto the microtubule', Biochem. Biophys. Res. Commun. Vol. 350, No. 4, pp. $850-853$.

23. Mootha, V.K., Bunkenborg, J., Olsen, J.V., Hjerrild, M. et al. (2003), 'Integrated analysis of protein composition, tissue diversity, and gene regulation in mouse mitochondria', Cell Vol. 115, No. 5, pp. 629-640.

24. Cao, J., Xu, H., Zhao, H., Gong, W. et al. (2009), 'The mechanisms of human hotdog-fold thioesterase 2 (hTHEM2) substrate recognition and catalysis illuminated by a structure and function based analysis', Biochemistry Vol. 48, No. 6, pp. 1293-1304.

25. Wei, J., Kang, H.W. and Cohen, D.E. (2009), 'Thioesterase superfamily member 2 (Them2)/acyl-CoA thioesterase 13 (Acot13): A homotetrameric hotdog fold thioesterase with selectivity for long-chain fatty acyl-CoAs', Biochem. J. Vol. 421, pp. 311-322.

26. Parcellier, A., Tintignac, L.A., Zhuravleva, E., Cron, P. et al. (2009), 'Carboxy-terminal modulator protein (CTMP) is a mitochondrial protein that sensitizes cells to apoptosis', Cell Signal. Vol. 21, pp. 639-650.

27. Piao, L., Li, Y., Yang, K.J., Park, K.A. et al. (2009), 'Heat shock protein 70-mediated sensitization of cells to apoptosis by carboxyl-terminal modulator protein', BMC Cell Biol. Vol. 10, p. 53.

28. Zhao, H., Martin, B.M., Bisoffi, M. and Dunaway-Mariano, D. (2009), 'The Akt C-terminal modulator protein is an acyl-CoA thioesterase of the Hotdog-Fold family', Biochemistry Vol. 48, No. 24, pp. 5507-5509. 